\title{
Interlayer-coupled spin vortex pairs and their response to external magnetic fields
}

\author{
Sebastian Wintz, ${ }^{1, *}$ Christopher Bunce, ${ }^{1, \dagger}$ Anja Banholzer, ${ }^{1}$ Michael Körner, ${ }^{1}$ Thomas Strache, ${ }^{1, \ddagger}$ Roland Mattheis, ${ }^{2}$ \\ Jeffrey McCord, ${ }^{3}$ Jörg Raabe, ${ }^{4}$ Christoph Quitmann, ${ }^{4}$ Artur Erbe,${ }^{1}$ and Jürgen Fassbender ${ }^{1}$ \\ ${ }^{1}$ Helmholtz-Zentrum Dresden-Rossendorf, 01328 Dresden, Germany \\ ${ }^{2}$ Institut für Photonische Technologien, 07702 Jena, Germany \\ ${ }^{3}$ Christian-Albrechts Universität zu Kiel, 24118 Kiel, Germany \\ ${ }^{4}$ Swiss Light Source, Paul Scherrer Institut, 5232 Villigen, Switzerland
}

(Received 16 March 2012; published 20 June 2012)

\begin{abstract}
We report on the response of multilayer spin textures to static magnetic fields. Coupled magnetic vortex pairs in trilayer elements (ferromagnetic/nonmagnetic/ferromagnetic) are imaged directly by means of layer-selective magnetic x-ray microscopy. We observe two different circulation configurations with parallel and opposing senses of magnetization rotation at remanence. Upon application of a field, all of the vortex pairs investigated react with a displacement of their cores. For purely dipolar coupled pairs, the individual core displacements are similar to those of an isolated single-layer vortex, but also a noticeable effect of the mutual stray fields is detected. Vortex pairs that are linked by an additional interlayer exchange coupling (IEC), which is either ferromagnetic or antiferromagnetic, mainly exhibit a layer-congruent response. We find that, apart from a possible decoupling at higher fields, these strict IEC vortex pairs can be described by a single-layer model with effective material parameters. This result implies the possibility to design multilayer spin structures with arbitrary effective magnetization.
\end{abstract}

DOI: 10.1103/PhysRevB.85.224420

PACS number(s): 75.70.Kw, 75.75.-c, 75.70.Cn, 68.37.Yz

\section{INTRODUCTION}

Micromagnetic spin structures have been investigated for many years. ${ }^{1,2}$ In particular, objects with nontrivial topologies such as skyrmion crystals ${ }^{3,4}$ or spin vortices ${ }^{5-12}$ have attracted significant attention. Spin vortices typically occur in micronsized ferromagnetic thin film elements ${ }^{13}$ and, as sketched in Fig. 1(a), they consist of a planar flux-closing magnetization (M) curl that turns out of the plane in the very small central core region $(\sim 10 \mathrm{~nm}) .{ }^{1,5,14}$ The curl's sense of rotation (circulation $c$ ) as well as the orientation of the core (polarity $p$ ) can be quantified to

$$
\begin{gathered}
c=\operatorname{sgn}\left\{\mathbf{e}_{z} \cdot[\mathbf{r} \times \mathbf{M}(r \neq 0)]\right\}, \\
p=\operatorname{sgn}\left[\mathbf{e}_{z} \cdot \mathbf{M}(r=0)\right]
\end{gathered}
$$

if a central core at $r=0$ is assumed. Since $c$ and $p$ can independently be in either the +1 or -1 state, vortices are chiral objects that occur with two opposing handednesses $(h=c \cdot p)$. These configurations are reflected in the various dynamic eigenmodes of a spin vortex. For instance, the sense of the prominent core gyration mode is determined by $p^{6,7,11,15}$ Motivated by the numerous studies on single-layer vortices, proposals were made for their application as memory cells or spin-transfer torque-driven oscillators. ${ }^{16-20}$

From the viewpoint of these concepts, but also from a fundamental perspective, the coupling between spatially confined vortices is a key issue to address..$^{21,22}$ Especially, vortices that are coupled vertically via a nonmagnetic interlayer are of interest, ${ }^{23-34}$ as significant magnetoresistance and spin-torque phenomena may occur. ${ }^{35-39}$ The relevant coupling mechanisms for such geometries are the magnetodipolar interaction and, depending on the spacer material and thickness, also interlayer exchange coupling (IEC). ${ }^{40-43}$ As sketched in Fig. 1(b), four basic vortex pair configurations with respect to the combination $(C, P)$ of $C=\prod_{i} c_{i}$ and $P=\prod_{i} p_{i}$ exist. The curl's can be either oriented ferromagnetically (FM: $C=1$ ) or antiferromagnetically (AF: $C=-1$ ), whereas the cores are either aligned parallel (PL: $P=1$ ) or antiparallel (AP: $P=-1$ ). Again, each of the four states shown in Fig. 1(b) has a counterpart with opposing handedness. In the case of the square-shaped elements sketched, flux closure is realized by the formation of four magnetic domains, which are interconnected by $90^{\circ}$ domain walls. As these discrete vortices have the same topology as continuous (circular) ones, we will refer to both of them as vortices in the following text.

The reports available on interlayer-coupled vortices can be categorized into static investigations ${ }^{23,24,26-29}$ on the one hand and magnetization dynamics studies using external fields ${ }^{30-34}$ or spin-polarized currents s $^{35-39}$ as excitation sources on the other hand. Many of the approaches used to analyze the system are theoretical, ${ }^{30-33,39}$ whereas the experimental approaches are often based on integral resistance measurements, ${ }^{35-38}$ or rely on information averaged from an ensemble of structures. ${ }^{26-29}$ Only Refs. 23-25 and 34 address the microscopic properties of individual stacked vortex pairs by means of a direct, layer-selective imaging technique. Among these, Chou et al. ${ }^{34}$ and $\mathrm{Wu}$ et $a .^{23}$ focus on FM vortex pair configurations, and also the existence of AF states has been shown recently. ${ }^{24}$ In addition, it was found that these $C$ states can be predetermined by a control of IEC..$^{24,25}$ In this article, we will report on the response of individual vortex pairs (which are imaged directly by soft x-ray microscopy) to the application of quasistatic magnetic fields. We will discuss the behavior of different $C$ states for pure dipolar coupling (PDC) as well as IEC. An effective single-layer model will be presented to describe the case of strict (layer-congruent) vortex coupling. 


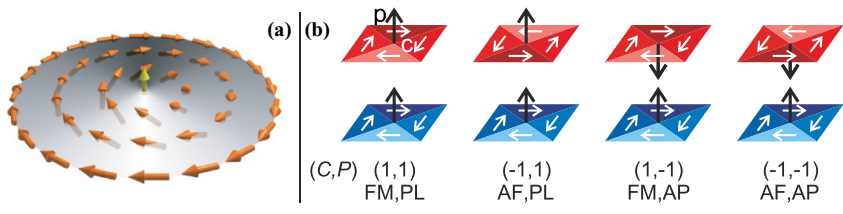

FIG. 1. (Color online) Vortex configurations in magnetic thin films. (a) Circular single-layer disk, where arrows are indicating the direction of M. Both the sense of the planar magnetization curl (circulation $c$ ) and the orientation of the central core (polarity $p$ ) can independently be either in the +1 or -1 state. (b) Four basic configurations of a stacked vortex pair with respect to the relative orientations of $c$ (FM or $\mathrm{AF})$ and $p(\mathrm{PL}$ or $\mathrm{AP})$ in a square-shaped element.

\section{EXPERIMENTAL DETAILS}

\section{A. Sample preparation}

Circular disks (circles) and square-shaped elements (squares) with a lateral size of $2 \mu \mathrm{m}$ were fabricated in order to prepare vortex pair states in trilayer structures. The creation of the patterned elements was accomplished by a sequence of electron beam lithography, multilayer deposition, and lift-off processing. Additionally, a $\mathrm{Cu}$ stripline of $150 \mathrm{~nm}$ thickness $(t)$ was fabricated on top of these elements for use as an excitation source in other experiments. As specified in Table I, six different multilayer stacks were used for the patterned elements in order to investigate PDC and IEC vortex pairs. 11 of the functional trilayer films used in this work consist of two ferromagnetic layers, which are separated by a nonmagnetic interlayer. The films containing a $\mathrm{Ru}$ or $\mathrm{Cu}$ interlayer were deposited by means of dc magnetron sputtering, whereas electron beam evaporation was used to grow the films with $\mathrm{Rh}$ spacer. X-ray transparent silicon-nitride ( $\mathrm{SiN})$ membranes with $t=100 \mathrm{~nm}$ serve as deposition substrates. Al or $\mathrm{Cr}$ capping layers protect the stacks from oxidation. Co and permalloy $\left(\mathrm{Ni}_{81} \mathrm{Fe}_{19}\right)$ were used as ferromagnetic layers. This allows for a layer-selective detection via $\mathrm{x}$-ray absorption spectroscopy. The reason for using different spacer materials and thicknesses is to create a strong antiferromagnetic (afm) IEC on the one hand $(\mathrm{Ru}, \mathrm{Rh} ; t=0.8 \mathrm{~nm}),{ }^{40}$ and a PDC with no IEC contribution on the other hand $(\mathrm{Cu} ; t=10 \mathrm{~nm})$. The thicknesses chosen for the ferromagnetic layers are expected to enforce the formation of vortex pair states in micron-sized elements. ${ }^{24,25,44}$

In order to generate vortex pairs with ferromagnetic (fm) IEC, we made use of the ion-induced IEC modification, which

TABLE I. Naming and composition of the functional trilayer stacks. The thicknesses given are nominal (regular font) or as inferred from cross-sectional transmission electron microscopy (italic font).

\begin{tabular}{lc}
\hline \hline Sample & Composition $[t(\mathrm{~nm})]$ \\
\hline $\mathrm{Co} / \mathrm{Cu} / \mathrm{NiFe}(50)$ & $\mathrm{SiN} / \mathrm{Co}(48) / \mathrm{Cu}(10) / \mathrm{Ni}_{81} \mathrm{Fe}_{19}(42) / \mathrm{Al}(5)$ \\
$\mathrm{Co} / \mathrm{Cu} / \mathrm{NiFe}(25)$ & $\mathrm{SiN} / \mathrm{Co}(24) / \mathrm{Cu}(10) / \mathrm{Ni}_{81} \mathrm{Fe}_{19}(21) / \mathrm{Al}(5)$ \\
$\mathrm{Co} / \mathrm{Ru} / \mathrm{NiFe}(50)$ & $\mathrm{SiN} / \mathrm{Co}(48) / \mathrm{Ru}(0.8) / \mathrm{Ni}_{81} \mathrm{Fe}_{19}(42) / \mathrm{Al}(5)$ \\
$\mathrm{Co} / \mathrm{Ru} / \mathrm{NiFe}(25)$ & $\mathrm{SiN} / \mathrm{Co}(24) / \mathrm{Ru}(0.8) / \mathrm{Ni}_{81} \mathrm{Fe}_{19}(21) / \mathrm{Al}(5)$ \\
$\mathrm{Co} / \mathrm{Rh} / \mathrm{NiFe}(50)$ & $\mathrm{SiN} / \mathrm{Co}(50) / \mathrm{Rh}(0.8) / \mathrm{Ni}_{{ }_{11}} \mathrm{Fe}_{19}(50) / \mathrm{Cr}(3)$ \\
$\mathrm{Co} / \mathrm{Rh} / \mathrm{NiFe}(25)$ & $\mathrm{SiN} / \mathrm{Co}(25) / \mathrm{Rh}(0.8) / \mathrm{Ni}_{81} \mathrm{Fe}_{19}(25) / \mathrm{Cr}(3)$ \\
\hline \hline
\end{tabular}

TABLE II. Bilinear $\left(J_{\mathrm{L}}\right)$ and biquadratic $\left(J_{\mathrm{Q}}\right)$ IEC constants for different trilayer stacks.

\begin{tabular}{lccc}
\hline \hline Sample & $\begin{array}{c}\mathrm{Ne}^{+} \\
\left(10^{15} \mathrm{~cm}^{-2}\right)\end{array}$ & $\begin{array}{c}J_{\mathrm{L}} \\
\left(\mathrm{mJ} / \mathrm{m}^{2}\right)\end{array}$ & $\begin{array}{c}J_{\mathrm{Q}} \\
\left(\mathrm{mJ} / \mathrm{m}^{2}\right)\end{array}$ \\
\hline $\mathrm{Co} / \mathrm{Cu} / \mathrm{NiFe}(25)$ & 0 & 0 & 0 \\
$\mathrm{Co} / \mathrm{Ru} / \mathrm{NiFe}(25)$ & 0 & -0.35 & -0.07 \\
$\mathrm{Co} / \mathrm{Rh} / \mathrm{NiFe}(25): \mathrm{Ne}^{+} 0.3$ & 0.3 & 0.85 & 0 \\
$\mathrm{Co} / \mathrm{Rh} / \mathrm{NiFe}(25): \mathrm{Ne}^{+} 2.0$ & 2 & 1.1 & 0 \\
\hline \hline
\end{tabular}

had been pioneered by Demokritov et al. ${ }^{45}$ Irradiation of afm IEC disks with $\mathrm{Ne}$ ions results in a reorientation of the coupling from afm to $\mathrm{fm}$ and by this the $C$ configuration of a vortex pair changes from AF to FM. ${ }^{25}$ Empirically, IEC can be quantified by its bilinear $\left(J_{\mathrm{L}}\right)$ and biquadratic $\left(J_{\mathrm{Q}}\right)$ coupling constants with respect to the areal energy density $\left(\sigma_{\text {IEC }}\right)$ :

$$
\sigma_{\mathrm{IEC}}=-J_{\mathrm{L}} \frac{\mathbf{M}_{1} \cdot \mathbf{M}_{2}}{M_{1} M_{2}}-J_{\mathrm{Q}} \frac{\left(\mathbf{M}_{1} \cdot \mathbf{M}_{2}\right)^{2}}{\left(M_{1} M_{2}\right)^{2}},
$$

where $\mathbf{M}_{i}$ is the magnetization vector of the $i$ th layer. ${ }^{46}$ Hence, a negative $J_{\mathrm{L}}(\mathrm{afm})$ favors an antiparallel orientation of the ferromagnetic layers and vice versa $\left[J_{\mathrm{L}}>0(\mathrm{fm})\right.$ $\Rightarrow$ parallel]. A contribution for a perpendicular alignment is induced by $J_{\mathrm{Q}} \neq 0$. Details about the measurement of the coupling constants and the IEC modification by $\mathrm{Ne}$ irradiation with respect to the systems used in this work can be found in Ref. 25. In Table II, $J_{\mathrm{L}}$ and $J_{\mathrm{Q}}$ are given for the as-prepared $\mathrm{Co} / \mathrm{Cu} / \mathrm{NiFe}(25)$ and $\mathrm{Co} / \mathrm{Ru} / \mathrm{NiFe}(25)$ stacks as well as for $\mathrm{Co} / \mathrm{Rh} / \mathrm{NiFe}(25)$ films that were irradiated with $60-\mathrm{keV} \mathrm{Ne}$ ions. Obviously, the $\mathrm{Co} / \mathrm{Cu} / \mathrm{NiFe}$ stacks are $\mathrm{PDC}$, the unirradiated $\mathrm{Co} / \mathrm{Ru} / \mathrm{NiFe}$ stacks are afm IEC, and the $\mathrm{Co} / \mathrm{Rh} / \mathrm{NiFe}$ stacks with a Ne irradiation fluence beyond $0.3 \times 10^{15} \mathrm{~cm}^{-2}$ are fm IEC. As shown later, for the irradiated stacks, the coupling type of the thicker $\mathrm{Co} / . . . \mathrm{NiFe}(50)$ stacks is equivalent to the corresponding $\mathrm{Co} / . . . \mathrm{NiFe}(25)$ ones when the $\mathrm{Ne}$ energy is increased to $110 \mathrm{kV}$. Furthermore, the coupling constants are assumed to be equal, as the interlayer thickness is kept constant and the interface roughness does not significantly change from the $\mathrm{Co} / . . . \mathrm{NiFe}(25)$ to the $\mathrm{Co} / . . . / \mathrm{NiFe}(50)$ samples. Note that as this article focuses on comparing vortex pairs with fm and afm IEC, we do not discuss unirradiated $\mathrm{Co} / \mathrm{Rh} / \mathrm{NiFe}$ stacks since they exhibit a significant biquadratic coupling contribution.

\section{B. Magnetic x-ray microscopy}

Soft $x$-ray magnetic microscopy ${ }^{47,48}$ was used to image the local magnetic configuration in the coupled trilayer elements upon the application of static magnetic fields. In the realization of a scanning transmission x-ray microscope (STXM), an element-selective detection of the magnetization orientation with a lateral resolution below $25 \mathrm{~nm}$ is possible. ${ }^{25,49,50}$ X-ray magnetic circular dichroism (XMCD), which occurs for circularly polarized $\mathrm{x}$ rays at the $L$-absorption edges serves as contrast effect for this. ${ }^{51}$ The XMCD is proportional to the projection of the magnetization orientation $\mathbf{m}=\mathbf{M} / M$ onto the photon propagation direction. To be sensitive to an in-plane magnetization component, the sample is rotated by $30^{\circ}$ with respect to the incident $\mathrm{x}$-ray beam. The normalized 
difference of the signal taken from both opposing circular polarizations leads to a pure magnetic contrast. The ability to do layer-selective imaging stems from measuring the sample at two different photon energies corresponding to the $\mathrm{Ni}$ $L_{3}$-absorption edge $(\approx 853 \mathrm{eV})$ and to the Co $L_{3}$-absorption edge $(\approx 778 \mathrm{eV})$, respectively. Only the layer that contains the specific element contributes to the dichroic absorption.

\section{Micromagnetic simulations}

In order to compare the experimental results with theoretical predictions and to evaluate a simplified single-layer model of the system (which applies when the vortex coupling is strict), three-dimensional micromagnetic simulations were undertaken. ${ }^{52}$ Trilayer circles and squares with sizes and parameters in accordance with the experimentally determined values $^{53}$ were simulated on a $256 \times 256 \times(1+1+1)$ grid, while for the effective single-layer model a $256 \times 256 \times 1$ grid was used. The initial magnetization states were defined to match those observed in the experiment. Stationary states were accessed by integrating the Landau-Lifshitz-Gilbert equation ${ }^{54}$ with a high damping coefficient $(\alpha=1)$.

\section{RESULTS AND DISCUSSION}

\section{A. Response to static magnetic fields}

We investigated the response of interlayer-coupled vortices in trilayer disks to quasistatic magnetic fields. STXM was used to directly image the planar magnetization distribution $\left(\sim M_{x}\right)$ in the $\mathrm{NiFe}$ and Co layer separately. Figure 2 shows

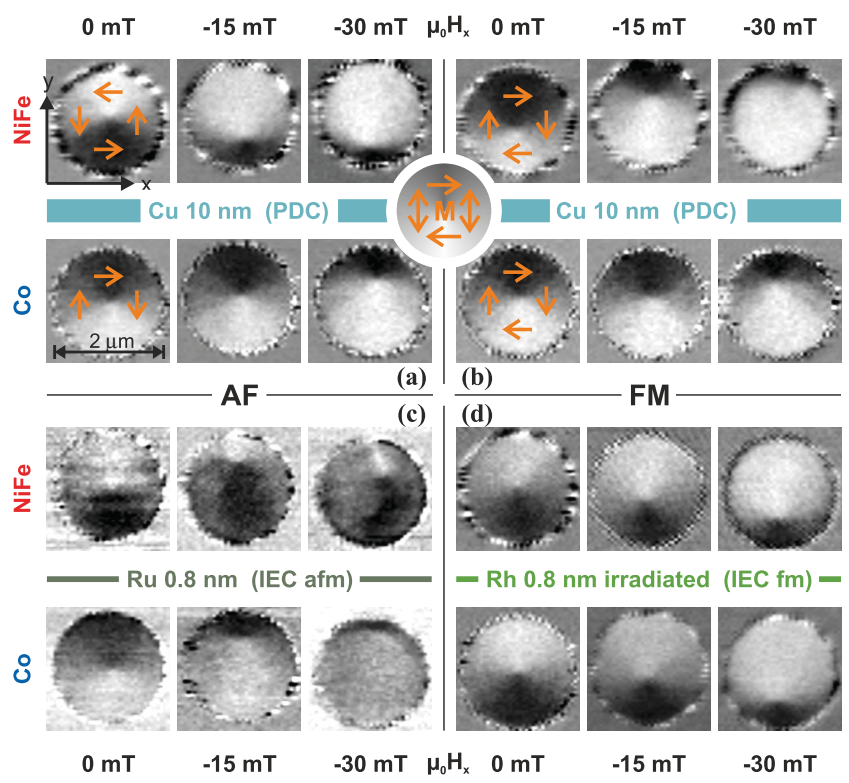

FIG. 2. (Color online) Response of vortex pairs in trilayer disks to external magnetic fields. STXM images showing the layer-specific magnetic orientation (in plane) for different fields applied into the $-x$ direction. The contrast mechanism is sensitive to the $M_{\mathrm{x}}$ component as indicated by the arrows. (a), (b) $\mathrm{PDC} \mathrm{Co} / \mathrm{Cu} / \mathrm{NiFe}(50)$ disks with (a) $\mathrm{AF}$ and (b) FM configuration at remanence. (c) $\mathrm{Co} / \mathrm{Ru} / \mathrm{NiFe}(50)$ afm IEC disk in AF state, (d) irradiated $\mathrm{Co} / \mathrm{Ru} / \mathrm{NiFe}(50): \mathrm{Ne}^{+} 0.3 \mathrm{fm}$ IEC disk configured FM. the micrographs of four different vortex pairs at remanence and upon application of a field: AF [Figs. 2(a) and 2(c)] and FM [Figs. 2(b) and 2(d)] states in PDC [Figs. 2(a) and 2(b)] and IEC [Figs. 2(c) and 2(d)] disks, respectively. External magnetic fields of $\mu_{0} H_{x}=-15$ and $-30 \mathrm{mT}$ were applied to the samples. The orientation of the applied field is parallel to the magnetization of the bright regions in the STXM images. In agreement with recent studies, ${ }^{24}$ both $C$ configurations are present in $\mathrm{PDC}$ disks of $\mathrm{Co} / \mathrm{Cu} / \mathrm{NiFe}(50)$ [cf. Figs. 2(a) and 2(b)]. In both cases, the vortices of the individual layers respond upon application of a field by a displacement of their core positions $r_{\mathrm{c}}=\left(x_{\mathrm{c}}, y_{\mathrm{c}}\right)$. For the AF state, the cores are displaced in opposite directions (a) $(\mathrm{Co}:+y / \mathrm{NiFe}:-y)$, while for the FM configuration they move in the same direction $(+y)$. The distance moved increases with the applied field. However, the core displacement in the NiFe layer is always bigger than that in the Co layer. At $\mu_{0} H_{x}=-30 \mathrm{mT}$, the FM pair exhibits a small $x$ displacement of the cores into opposite directions $(\mathrm{Co}:-x, \mathrm{NiFe}:+x)$ that is superimposed onto the $y$ displacement. Also, in this case the core displacement magnitude in the NiFe layer exceeds that in the Co layer.

This behavior can be understood on the basis of the response of an isolated single-layer vortex to an applied field. Such a single-layer vortex would exhibit a core displacement along the planar axis perpendicular to the field. ${ }^{55}$ The direction of displacement corresponds to the $c$ state in the way that the sample area which has its magnetization pointing parallel to the applied field is enlarged. This reduces the Zeeman energy

$$
E_{\text {Zem }}=-\mu_{0} \int^{V} \mathbf{H} \cdot \mathbf{M} d^{3} r,
$$

where $\mathbf{H}$ is the external magnetic field. Based on this, we can explain the behavior of the individual vortices in the PDC pairs as two quasiindependent responses. Regardless of the state of the Co layer, the NiFe core moves in a manner that increases the size of the bright sample region where the magnetization lies parallel to the applied field, a fact which vice versa holds true for the Co core. This response is in qualitative agreement with predictions that have been made for the AF configuration. ${ }^{28,29}$ Analog to single-layer vortices, ${ }^{55}$ the bigger displacement of the $\mathrm{NiFe}$ cores as compared to those in Co is a consequence of the different magnetic moment per unit area $\left(M_{\mathrm{s}} t\right)$. However, there are two unique features in the PDC response. As supported by micromagnetic simulations (not shown), the core shift of the individual vortices in the PDC pairs is smaller than that of the corresponding single layer. We attribute this effect to counteracting stray fields, which, in the case of the NiFe vortex, are generated by the shifted Co vortex and vice versa. The superimposed $x$ displacement at $\mu_{0} H_{x}=-30 \mathrm{mT}$ observed for the FM pair is also reproduced by simulations (not shown).

Figure 2(c) shows the response to a field of an AF vortex pair in a $\mathrm{Co} / \mathrm{Ru} / \mathrm{NiFe}(50)$ disk with afm IEC. At remanence, the cores in both layers are exactly on top of each other. Up to $\mu_{0} H_{x}=-15 \mathrm{mT}$, the cores are displaced congruently into the $+y$ direction. The reason for this is the relatively strong afm IEC $\left(J_{\mathrm{L}}=-0.35 \mathrm{~mJ} / \mathrm{m}^{2}\right)$. It forces the $\mathrm{NiFe}$ core to move with the Co core, even though this enlarges the dark regions in the $\mathrm{NiFe}$ vortex, which are oriented antiparallel to 
the field. The Co layer is determining the direction of the congruent core displacement, as its higher $M_{\mathrm{s}} t$ is dominating the net moment of the trilayer. At $\mu_{0} H_{x}=-30 \mathrm{mT}$, however, the Co core is located close to the upper sample edge, whereas the NiFe core has started to shift back into the $-y$ direction. Obviously, the IEC is no longer sufficient to enforce layer congruency against the disruptive $E_{\text {Zem }}$ contribution. Therefore, the vortices decouple at higher fields.

For the FM configuration in a $\mathrm{Co} / \mathrm{Ru} / \mathrm{NiFe}(50): \mathrm{Ne}^{+} 0.3$ stack with fm IEC [cf. Fig. 2(d)], we observed a layercongruent core shift into the $-y$ direction for all fields up to $\mu_{0} H_{x}=-30 \mathrm{mT}$. In contrast to the AF IEC pair, here the IEC is obviously strong enough to preserve the congruency. There are two main reasons for this: (i) the absolute value of the coupling constant $J_{\mathrm{L}}=0.85 \mathrm{~mJ} / \mathrm{m}^{2}$ is more than twice as high, and (ii) the external field does not energetically prefer to drive the cores into opposite directions. Also, the difference in $M_{\mathrm{s}} t$ between the two layers, which would favor a different shift distance, is not sufficient to cause a decoupling at the fields applied. This behavior is in qualitative agreement with previous predictions. ${ }^{26}$ Note that at $\mu_{0} H_{x}=-15 \mathrm{mT}$, the $y$ shift of the FM IEC pair is smaller than that of the corresponding AF pair.

In summary, we observed that the individual layers of a PDC pair, for both configurations $\mathrm{AF}$ and FM, behave in a similar manner to a single isolated vortex. However, the mutual stray fields are reducing the core displacements as compared to single-layer vortices. In the case of the FM pair, a shift of the cores into opposite directions along the field axis is superimposed. Both IEC pairs, AF and FM, exhibit layer-congruent core displacements up to intermediate fields, with the shift of the AF configuration being larger. This is remarkable as for the $\mathrm{AF}$ state, the $\mathrm{NiFe}$ vortex is dragged into the unfavored direction with respect to the external field. At higher fields, the AF vortex pair decouples, while for the FM configuration, IEC preserves layer congruency. Note that in this work we do not discuss different $P$ configurations as the influence of the vortex cores on the quasistatic response is negligible for the sample sizes and external fields given. ${ }^{56}$

\section{B. Effective model for strictly coupled vortices}

In the following, we will focus on the special case of strict vortex coupling, meaning that the IEC is sufficiently strong to preserve layer congruency for all external fields applied up to the vortex annihilation limit. In order to achieve this case even for pairs with an AF configuration, we reduced the thickness of the magnetic layers to $t=25 \mathrm{~nm}$. This is to change the ratio of the surface proportional $E_{\mathrm{IEC}}$ to the volume proportional $E_{\text {Zem }}$. Additionally, this $t$ reduction is expected to decrease the vortex annihilation fields. ${ }^{55}$ For the same reason (but also to demonstrate the general validity), we used $2-\mu \mathrm{m}$ squares here instead of disks.

Figure 3 shows the response of $2-\mu \mathrm{m}$-sized trilayer squares with strong fm IEC [Figs. 3(a) and 3(b)] and afm IEC [Figs. 3(c) and 3(d)], respectively. Each panel contains experimental STXM images and the results of micromagnetic simulations of an equivalent system. The top (bottom) rows of the figure correspond to the $\mathrm{NiFe}(\mathrm{Co})$ layer of the samples. The magnetic contrast is as depicted and described earlier. The external field ranges from 0 (remanence) to $\mu_{0} H_{x}=-30 \mathrm{mT}$. As for the disks, at remanence we observed a vortex pair with FM configuration [Figs. 3(a) and 3(b)] in the fm IEC sample and an AF state [Figs. 3(c) and 3(d)] in the afm IEC sample.

Figures 3(a) and 3(b) display the FM vortex pair in a $\mathrm{Co} / \mathrm{Rh} / \mathrm{NiFe}(25): \mathrm{Ne}^{+} 2.0$ square with $J_{\mathrm{L}}=1.1 \mathrm{~mJ} / \mathrm{m}^{2}$ separately for (a) the Co and (b) the NiFe layers. In the experiment, as well as the simulation, the vortices exhibit a strictly coupled response to the external field, i.e., a stepwise congruent core displacement into the $-y$ direction. At $\mu_{0} H_{x}=-30 \mathrm{mT}$, both vortices have vanished, which means that the cores were shifted out of the square. The core positions observed in the experiment are well reproduced by the simulations for the different fields. The explanation for the field-dependent core position of this FM IEC pair in a square is an analog of that in a disk [cf. Fig. 2(d)]. However, the reduced layer thickness and the altered geometry cause larger core displacements and the eventual core annihilation.

Figures 3(c) and 3(d) show the $\mathrm{NiFe}$ and the Co layers of the $\mathrm{AF}$ vortex state in a $\mathrm{Co} / \mathrm{Ru} / \mathrm{NiFe}(25)$ square with $J_{\mathrm{L}}=-0.35 \mathrm{~mJ} / \mathrm{m}^{2}$, respectively. Already at remanence, it is

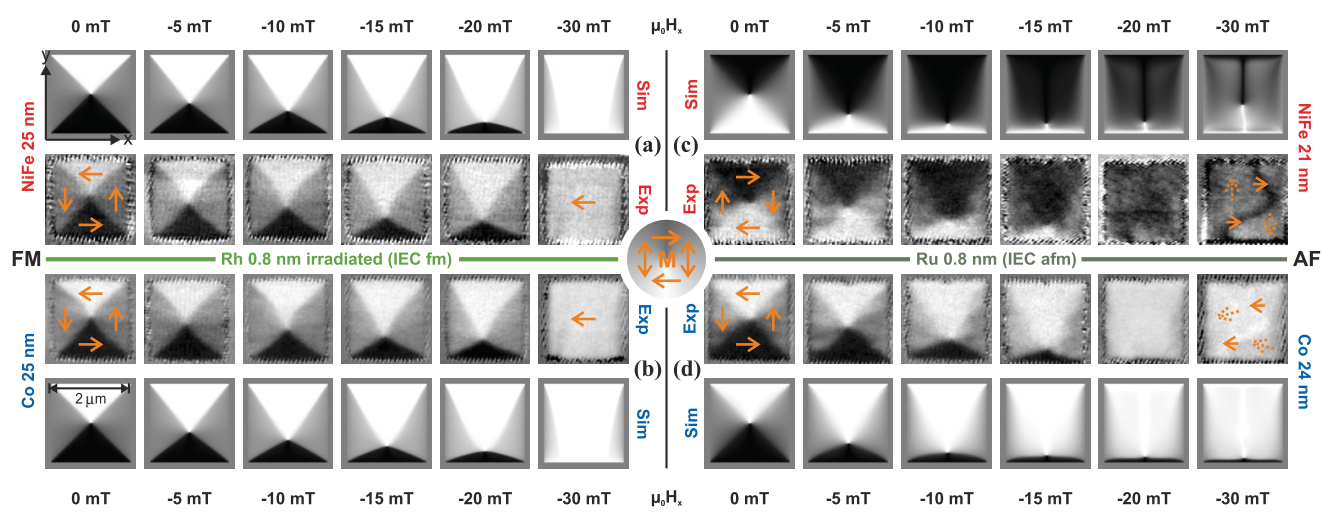

FIG. 3. (Color online) Response of strongly IEC coupled vortex pairs in trilayer squares to external magnetic fields. STXM images and micromagnetic simulations showing the layer-specific magnetic orientation (in plane) for different fields applied into the $-x$ direction. (a) NiFe and (b) Co vortex in an irradiated fm IEC Co/Rh/NiFe(25): $\mathrm{Ne}^{+} 2.0$ square. (c) $\mathrm{NiFe}$ and (d) Co vortex in an afm IEC Co/ Ru/ $\mathrm{NiFe}(25) \mathrm{square}$. Dotted arrows indicate the qualitative orientation of magnetization. 
noticeable that the width of the $90^{\circ}$ domain walls is wider than for the FM case, a fact that is in agreement with predictions made in Ref. 33. For external fields up to $\mu_{0} H_{x}=-15 \mathrm{mT}$, we experimentally observed a congruent core motion in both layers into the $-y$ direction. This corresponds to an enlargement of the bright domain (where $M$ is parallel to the applied field) in the Co layer and thus of the net moment of the trilayer. The response in this field range is comparable to that of the AF pair in the afm IEC disk [cf. Fig. 2(c)]. However, the displacements are again larger and, instead of vortex core decoupling, we here observed their annihilation at $\mu_{0} H_{x}=-20 \mathrm{mT}$. Again, this is a consequence of the reduced layer thickness and modified sample geometry. Compared to the FM configuration in the square, the cores of the AF state respond with a bigger displacement and they annihilate at smaller fields.

The experimental images at $\mu_{0} H_{x}=-30 \mathrm{mT}$ reveal for both layers a state with two domains that are separated by a meandering compensated Néel wall. ${ }^{2}$ The position of the wall is identical for the two layers and the magnetization in the wall is still antiparallel between both layers, with the Co layer pointing into the same direction like the field. In the NiFe layer, the domains adjacent to the wall are in the process of rotating into the field direction. This becomes obvious by the moderate contrast in the image and is also in agreement with the magnetization reversal of the corresponding continuous film (saturation field: $\mu_{0} H_{\mathrm{s}} \approx 45 \mathrm{mT}$ ). ${ }^{24}$ To compensate for this $\mathrm{NiFe}$ rotation, the domains in the Co also slightly tilt away from the field, which is indicated by the very weak image contrast.

The simulations are in good agreement with the experimental results for fields up to $\mu_{0} H_{x}=-15 \mathrm{mT}$. However, instead of a subsequent vortex annihilation event, the simulations predict a vortex decoupling for $\mu_{0} H_{x}=-20 \mathrm{mT}$ with the cores still being in the sample at $\mu_{0} H_{x}=-30 \mathrm{mT}$. In this point, the simulations show a response that is similar to the experimental results recorded for the AF pair in the afm IEC disk [cf. Fig. 2(c)]. The reason for the disagreement between the simulations and the experiment in case of the AF square presumably is the reduced annihilation barrier due to the edge properties of the real structures. Sloped sample edges as well as roughness effects may reduce the vortex nucleation and annihilation barriers. ${ }^{57}$ However, even states like the one with the meandering domain wall can be reproduced by the simulations if a proper initialization is used. The results (not shown) reveal that the domain rotation in the NiFe layer occurs with opposing sense, as indicated by the dotted arrows in Fig. 3(c).

This experimental observation of strictly coupled vortices for both $C$ configurations ( $\mathrm{AF}$ and $\mathrm{FM}$ ) inspires the application of a single-layer model with effective material parameters. If the magnetization changes congruently in both layers, we propose that the system can be described using an effective magnetization $\left(\mathbf{M}_{\text {eff }}\right)$ and an effective layer thickness $\left(t_{\text {eff }}\right)$ according to

$$
\begin{gathered}
\mathbf{M}_{\mathrm{eff}}=\frac{\sum_{i} \mathbf{M}_{i} t_{i}}{\sum_{i} t_{i}}=\frac{\mathbf{M}_{\mathrm{Co}} t_{\mathrm{Co}}+\mathbf{M}_{\mathrm{NiFe}} t_{\mathrm{NiFe}}}{t_{\mathrm{Co}}+t_{\mathrm{NiFe}}}, \\
t_{\mathrm{eff}}=\sum_{i} t_{i}=t_{\mathrm{Co}}+t_{\mathrm{NiFe}}
\end{gathered}
$$

TABLE III. Effective parameters for the single-layer model describing strictly coupled vortices in IEC $\mathrm{Co} / . . . / \mathrm{NiFe}(25)$ films.

\begin{tabular}{lccc}
\hline \hline & & $\begin{array}{c}M_{\text {eff }} \\
\left(10^{6} \mathrm{~A} / \mathrm{m}\right)\end{array}$ & $\begin{array}{c}t_{\text {eff }} \\
(\mathrm{nm})\end{array}$ \\
\hline $\mathrm{FM}$ & $\mathrm{Stack}$ & 0.92 & 50 \\
$\mathrm{AF}$ & $\mathrm{Co} / \mathrm{Rh} / \mathrm{NiFe}(25): \mathrm{Ne}^{+} 2.0$ & 0.33 & 45 \\
\hline \hline
\end{tabular}

which leads to the parameters given in Table III, taking also into account the ion-induced reduction of $M_{\mathrm{s}} .{ }^{25}$ On the basis of this single-layer model with effective material parameters, it becomes clear that the bigger core displacement observed in the AF pair, as compared to the FM configuration, is a consequence of the much lower $M_{\text {eff }}{ }^{55}$

To evaluate the model, we quantitatively investigate the vortex core displacements $\left|y_{\mathrm{c}}\right|$ as a function of the applied field $\mu_{0}\left|H_{x}\right|$ as shown in Fig. 4. We compare the core positions measured from the experimental data (filled symbols) with the trilayer simulations (hollow symbols) and the effective single-layer simulations ${ }^{58}$ ( $\times$ symbol), respectively. Blue symbols correspond to the Co layer and red symbols to the $\mathrm{NiFe}$ one. This comparison is made for both configurations FM [Figs. 4(a) and 4(b)] and AF [Figs. 4(c) and 4(d)], each in a square [Figs. 4(a) and 4(c)] and a circle [Figs. 4(b) and 4(d)].

For the FM square shown in Fig. 4(a), the experimental core displacements are in good agreement with the trilayer

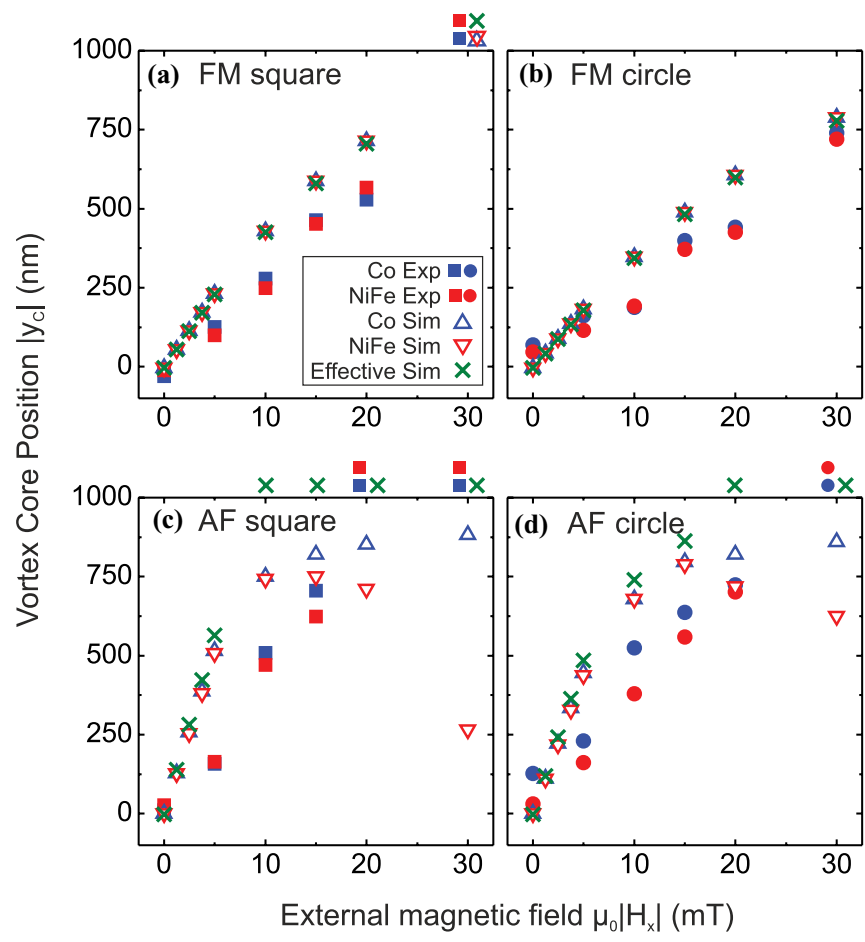

FIG. 4. (Color) Comparison of experimental (filled) and simulated (hollow) vortex core displacements in trilayer squares (squares) and disks (circles) upon the application of an external magnetic field $H_{x}$. In addition, the simulations are performed for a single-layer element with effective material parameters $(\times)$. Symbols positioned outside the graphs correspond to an annihilated vortex core. 
simulations as can also be seen in Fig. 3. The predicted core displacements are slightly too large, which probably stems from the uncertainty of the measured sample parameters $M_{\mathrm{s}}, t$ that were used as input for the simulations. This overestimation also occurs for the other three vortex pairs [Figs. 4(b), 4(c), and 4(d)]. In the experiment, as well as in the simulations, the cores of both layers remain on top of each other for all fields. If not stated otherwise, this fact also holds true for the other vortex pairs, taking into account the measurement accuracy given. Interestingly, the effective single-layer simulation perfectly matches the trilayer simulation, which strongly supports the validity of the effective single-layer model. Core annihilation occurs at $\mu_{0}\left|H_{x}\right|=30 \mathrm{mT}$ for all three cases. The FM circle shown in Fig. 4(b) exhibits a rather similar behavior to the FM square with respect to the experiment and the two simulation types. However, as for single-layer vortices, the core displacements in the circle are slightly smaller than in the square. This ensures that no annihilation occurs up to $\mu_{0}\left|H_{x}\right| \leqslant 30 \mathrm{mT}$. Both FM pairs exhibit core displacements that are almost proportional to the external field, especially up to $\mu_{0}\left|H_{x}\right| \leqslant 10 \mathrm{mT}$.

For the AF square shown in Fig. 4(c), a reasonable agreement between the experiment and the trilayer simulation exists up to $\mu_{0}\left|H_{x}\right| \leqslant 15 \mathrm{mT}$ (cf. Fig. 3). Above this field, the vortex cores annihilate in the experiment. However, in the trilayer simulation, they decouple with the $\mathrm{NiFe}$ core shifting back toward the center. This core decoupling already starts at $\mu_{0}\left|H_{x}\right|=15 \mathrm{mT}$, but only becomes significant at higher fields. The effective single-layer simulation agrees well with the trilayer simulation up to $\mu_{0}\left|H_{x}\right| \leqslant 5 \mathrm{mT}$, although the predicted single-layer shifts are slightly bigger than the trilayer ones. For intermediate fields $\mu_{0}\left|H_{x}\right|=10 \mathrm{mT}$, the effective single-layer simulation already predicts core annihilation, which is in disagreement with the trilayer simulation. Disagreement is also present for higher fields as the cores decouple in the trilayer simulation, a fact which violates the assumptions of the effective single-layer model. In the AF circle shown in Fig. 4(d), slightly smaller core displacements with respect to the AF square are exhibited as expected. The experimental core positions are in reasonable agreement with the trilayer simulations for $\mu_{0}\left|H_{x}\right| \leqslant 20 \mathrm{mT}$. However, at $\mu_{0}\left|H_{x}\right|=30 \mathrm{mT}$, the cores have annihilated in the experiment, whereas the trilayer simulations predict that they remain in the sample. In the trilayer simulations, the core decoupling starts at $\mu_{0}\left|H_{x}\right|=20 \mathrm{mT}$. Up to this field, the effective single-layer simulations agree well with the trilayer simulations. Again, the predicted shifts are slightly bigger in the single-layer simulation than in the trilayer simulation. Both AF pairs exhibit field-proportional shifts up to $\mu_{0}\left|H_{x}\right| \leqslant$ $5 \mathrm{mT}$. Above this field, the response decreases significantly. In the simulations, the AF configurations exhibit much bigger responses (square: $103 \mathrm{~nm} / \mathrm{mT}$, circle: $89 \mathrm{~nm} / \mathrm{mT}$ ) than the FM configurations (square: $43 \mathrm{~nm} / \mathrm{mT}$, circle: $35 \mathrm{~nm} / \mathrm{mT}$ ) over the range where the displacement is proportional to the field. Therefore, the core annihilations also occur at smaller fields.

The general agreement between the trilayer simulations and the single-layer simulations verifies that the single-layer model with effective parameters is well suited to describe the response of strictly coupled vortex pairs to quasistatic magnetic fields. In the framework of this model, the bigger core shifts of the AF pairs compared to the FM ones become evident. For the FM pair, there is an average $M_{\mathrm{s}}$, whereas for the AF configuration, the $M_{\mathrm{s}}$ 's subtract in a similar manner to a ferrimagnet.

\section{CONCLUSION}

We investigated the response of interlayer-coupled spin vortex pairs to static magnetic fields by direct STXM imaging. Both circulation configurations, FM and AF, were observed in circular as well as square-shaped trilayer elements. The interaction between the individual vortices is a combination of magnetodipolar coupling and IEC. All vortex pairs respond to planar field application with a displacement of their cores in order to reduce the emerging Zeeman energy.

In case of the PDC pairs, the individual vortices exhibit quasi-independent core displacements upon application of a field, similar to that of single vortices. Therefore, the cores move into the same direction when the configuration is FM, but into opposite directions when the pair is in an AF state. Also, the displacement in the $\mathrm{NiFe}$ layer is bigger than in the $\mathrm{Co}$ layer, due to the difference in $M_{\mathrm{s}} t .{ }^{55}$ However, the response in each layer is slightly reduced (as compared to the single-layer case) due to the counteracting stray fields generated by the core shift in each the other layer.

In the IEC samples, we observed a layer-congruent core displacement for both the FM and AF states up to intermediate fields, with the the Co circulation determining the direction of movement. The AF pair exhibits a significantly bigger core shift than the FM configuration. At higher fields, either the cores decouple or layer congruency persists up to annihilation. This is a consequence of three factors: (i) the field at which the collective annihilation would occur, (ii) the $C$ state (AF configurations tend to decouple more easily), and (iii) the ratio between the surface-dependent $E_{\mathrm{IEC}}$ and the volumedependent $E_{\text {Zem }}$ induced by the field. For strictly coupled vortices, i.e., layer congruency persists, the system can be described as a single-layer vortex with effective material parameters $\left(M_{\mathrm{eff}}, t_{\mathrm{eff}}\right)$. This result was verified by comparing the experimentally determined core displacements with trilayer simulations and effective single-layer simulations. In the framework of this single-layer model, the subtractive (AF) or averaging (FM) contribution to $M_{\text {eff }}$ explains the significant difference in core shift.

The results presented are of interest for the field of coupled spin systems, particularly interacting magnetic vortices. The results might also be relevant for possible application concepts involving interlayer-coupled vortices, such as memory cells or spin-torque oscillators. For example, the single-layer model with effective parameters verified here allows the design of "ferrimagnetic" IEC AF vortex pairs with an $M_{\text {eff }}$ that can be chosen to lie anywhere in the range of $0<M_{\text {eff }}<M_{\mathrm{s}}$ by varying the individual $M_{i} t_{i}$ 's. An interesting remaining question is whether the effective single-layer model is also valid for the magnetic-field-driven dynamic regime including core gyration and spin-wave modes, a question that could be answered in future studies involving time-resolved but also $p$-sensitive measurements. 


\section{ACKNOWLEDGMENTS}

We would like to thank A. Puzić for his contribution to this work as well as S. Gemming for fruitful discussions. We are grateful to V. Kühn (lithography), T. Schönherr, A. Neubert, U. Wiesenhütter (STXM), and K. Lenz (FMR) for their support. The STXM experi- ments were performed using the X07DA (PolLux) beamline at the Swiss Light Source, Paul Scherrer Institut, Villigen, Switzerland. Funding from BMBF (Contracts No. 05 KS4WE1/6 and No. FKZ 13N10144), DFG (Grant No. FA 314-7/1), and EU (Grant No. 226716) is acknowledged. *s.wintz@hzdr.de

${ }^{\dagger}$ Present address: 01326 Dresden, Germany.

${ }_{\ddagger}^{\ddagger}$ Present address: Vacuumschmelze GmbH \& Co. KG, 63450 Hanau, Germany.

${ }^{1}$ C. Kittel, Rev. Mod. Phys. 21, 541 (1949).

${ }^{2}$ A. Hubert and R. Schäfer, Magnetic Domains (Springer, Berlin, 2008).

${ }^{3}$ U. K. Rössler, A. N. Bogdanov, and C. Pfleiderer, Nature (London) 442, 797 (2006).

${ }^{4}$ S. Mühlbauer, B. Binz, F. Jonietz, C. Pfleiderer, A. Rosch, A. Neubauer, R. Georgii, and P. Böni, Science 323, 915 (2009).

${ }^{5}$ E. Feldtkeller and H. Thomas, Phys. Kondens. Mater. 4, 8 (1965).

${ }^{6}$ A. A. Thiele, Phys. Rev. Lett. 30, 230 (1973).

${ }^{7}$ D. L. Huber, J. Appl. Phys. 53, 1899 (1982).

${ }^{8}$ B. E. Argyle, E. Terrenzio, and J. C. Slonczewski, Phys. Rev. Lett. 53, 190 (1984).

${ }^{9}$ T. Shinjo, T. Okuno, R. Hassdorf, K. Shigeto, and T. Ono, Science 289, 930 (2000)

${ }^{10}$ J. Raabe, R. Pulwey, R. Sattler, T. Schweinbök, J. Zweck, and D. Weiss, J. Appl. Phys. 88, 4437 (2000).

${ }^{11}$ S.-B. Choe, Y. Acremann, A. Scholl, A. Bauer, A. Doran, J. Stöhr, and H. A. Padmore, Science 304, 420 (2004).

${ }^{12}$ B. Van Waeyenberge, A. Puzic, H. Stoll, K. W. Chou, T. Tyliszczak, R. Hertel, M. Fähnle, H. Brückl, K. Rott, G. Reiss, I. Neudecker, D. Weiss, C. H. Back, and G. Schütz, Nature (London) 444, 461 (2006).

${ }^{13}$ R. P. Cowburn, D. K. Koltsov, A. O. Adeyeye, M. E. Welland, and D. M. Tricker, Phys. Rev. Lett. 83, 1042 (1999).

${ }^{14}$ A. Wachowiak, J. Wiebe, M. Bode, O. Pietzsch, M. Morgenstern, and R. Wiesendanger, Science 298, 577 (2002).

${ }^{15}$ K. W. Chou, A. Puzic, H. Stoll, D. Dolgos, G. Schütz, B. Van Waeyenberge, A. Vansteenkiste, T. Tyliszczak, G. Woltersdorf, and C. H. Back, Appl. Phys. Lett. 90, 202505 (2007).

${ }^{16}$ A. Ruotolo, V. Cros, B. Georges, A. Dussaux, J. Grollier, C. Deranlot, R. Guillemet, K. Bouzehouane, S. Fusil, and A. Fert, Nat. Nanotechnol. 4, 528 (2009).

${ }^{17}$ V. S. Pribiag, I. N. Krivorotov, G. D. Fuchs, P. M. Braganca, O. Ozatay, J. C. Sankey, D. C. Ralph, and R. A. Buhrman, Nat. Phys. 3, 498 (2007).

${ }^{18}$ B. Pigeau, G. de Loubens, O. Klein, A. Riegler, F. Lochner, G. Schmidt, L. W. Molenkamp, V. S. Tiberkevich, and A. N. Slavin, Appl. Phys. Lett. 96, 132506 (2010).

${ }^{19}$ V. Sluka, A. Kákay, A. M. Deac, D. E. Bürgler, R. Hertel, and C. M. Schneider, J. Phys. D: Appl. Phys. 44, 384002 (2011).

${ }^{20}$ A. Dussaux, B. Georges, J. Grollier, V. Cros, A. V. Khvalkovskiy, A. Fukushima, M. Konoto, H. Kubota, K. Yakushiji, S. Yuasa, K. A. Zvezdin, K. Ando, and A. Fert, Nat. Commun. 1, 8 (2010).

${ }^{21}$ K. S. Buchanan, P. E. Roy, M. Grimsditch, F. Y. Fradin, K. Y. Guslienko, S. D. Bader, and V. Novosad, Nat. Phys. 1, 172 (2005).
${ }^{22}$ H. Jung, K.-S. Lee, D.-E. Jeong, Y.-S. Choi, Y.-S. Yu, D.-S. Han, A. Vogel, L. Bocklage, G. Meier, M.-Y. Im, P. Fischer, and S.-K. Kim, Sci. Rep. 1, 10 (2011).

${ }^{23}$ J. Wu, D. Carlton, E. Oelker, J. S. Park, E. Jin, E. Arenholz, A. Scholl, C. Hwang, J. Bokor, and Z. Q. Qiu, J. Phys.: Condens. Matter 22, 342001 (2010).

${ }^{24}$ S. Wintz, T. Strache, M. Körner, M. Fritzsche, D. Markó, I. Mönch, R. Mattheis, J. Raabe, C. Quitmann, J. McCord, A. Erbe, and J. Fassbender, Appl. Phys. Lett. 98, 232511 (2011).

${ }^{25}$ S. Wintz, T. Strache, M. Körner, C. Bunce, A. Banholzer, I. Mönch, R. Mattheis, J. Raabe, C. Quitmann, J. McCord, A. Erbe, K. Lenz, and J. Fassbender, Phys. Rev. B 85, 134417 (2012).

${ }^{26}$ P. Vavassori, V. Bonanni, A. Busato, D. Bisero, G. Gubbiotti, A. O. Adeyeye, S. Goolaup, N. Singh, C. Spezzani, and M. Sacchi, J. Phys. D: Appl. Phys. 41, 134014 (2008).

${ }^{27}$ E. Girgis, S. P. Pogossian, and M. Gbordzoe, J. Appl. Phys. 99, 014307 (2006).

${ }^{28}$ K. S. Buchanan, K. Y. Guslienko, A. Doran, A. Scholl, S. D. Bader, and V. Novosad, Phys. Rev. B 72, 134415 (2005).

${ }^{29}$ K. S. Buchanan, K. Y. Guslienko, S.-B. Choe, A. Doran, A. Scholl, S. D. Bader, and V. Novosad, J. Appl. Phys. 97, 10 H503 (2005).

${ }^{30}$ D. K. Schreiber, O. G. Heinonen, and A. K. Petford-Long, Phys. Rev. B 80, 014411 (2009).

${ }^{31}$ S.-H. Jun, J.-H. Shim, S.-K. Oh, S.-C. Yu, D.-H. Kim, B. Mesler, and P. Fischer, Appl. Phys. Lett. 95, 142509 (2009).

${ }^{32}$ K. Y. Guslienko, K. S. Buchanan, S. D. Bader, and V. Novosad, Appl. Phys. Lett. 86, 223112 (2005).

${ }^{33}$ D. V. Berkov and N. L. Gorn, J. Appl. Phys. 103, 053908 (2008).

${ }^{34}$ K. W. Chou, A. Puzic, H. Stoll, G. Schütz, B. Van Waeyenberge, T. Tyliszczak, K. Rott, G. Reiss, H. Brückl, I. Neudecker, D. Weiss, and C. H. Back, J. Appl. Phys. 99, 08F305 (2006).

${ }^{35}$ N. Wang, X. L. Wang, W. Qin, S. H. Yeung, D. T. K. Kwok, H. F. Wong, Q. Xue, P. K. Chu, C. W. Leung, and A. Ruotolo, Appl. Phys. Lett. 98, 242506 (2011).

${ }^{36}$ N. Locatelli, V. V. Naletov, J. Grollier, G. de Loubens, V. Cros, C. Deranlot, C. Ulysse, G. Faini, O. Klein, and A. Fert, Appl. Phys. Lett. 98, 062501 (2011).

${ }^{37}$ M. Kuepferling, C. Serpico, M. Pufall, W. Rippard, T. Mitchell Wallis, A. Imtiaz, P. Krivosik, M. Pasquale, and P. Kabos, Appl. Phys. Lett. 96, 252507 (2010).

${ }^{38}$ S. S. Cherepov, A. Konovalenko, V. Korenivski, and D. C. Worledge, IEEE Trans. Magn. 46, 2124 (2010).

${ }^{39}$ B. C. Choi, J. Rudge, E. Girgis, J. Kolthammer, Y. K. Hong, and A. Lyle, Appl. Phys. Lett. 91, 022501 (2007).

${ }^{40}$ S. S. P. Parkin, R. Bhadra, and K. P. Roche, Phys. Rev. Lett. 66, 2152 (1991).

${ }^{41}$ S. S. P. Parkin, Phys. Rev. Lett. 67, 3598 (1991).

${ }^{42}$ P. Grünberg, R. Schreiber, Y. Pang, M. B. Brodsky, and H. Sowers, Phys. Rev. Lett. 57, 2442 (1986). 
${ }^{43}$ R. S. Beach, J. McCord, P. Webb, and D. Mauri, Appl. Phys. Lett. 80, 4576 (2002).

${ }^{44}$ K. Y. Guslienko and K. L. Metlov, Phys. Rev. B 63, 100403(R) (2001).

${ }^{45}$ S. O. Demokritov, C. Bayer, S. Poppe, M. Rickart, J. Fassbender, B. Hillebrands, D. I. Kholin, N. M. Kreines, and O. M. Liedke, Phys. Rev. Lett. 90, 097201 (2003).

${ }^{46}$ M. Rührig, R. Schäfer, A. Hubert, R. Mosler, J. A. Wolf, S. Demokritov, and P. Grünberg, Phys. Status Solidi A 125, 635 (1991).

${ }^{47}$ J. Stöhr, Y. Wu, B. Hermsmeier, M. Samant, G. Harp, S. Koranda, D. Dunham, and B. Tonner, Science 259, 658 (1993).

${ }^{48}$ P. Fischer, G. Schütz, G. Schmahl, P. Guttmann, and D. Raasch, Z. Phys. B: Condens. Matter 101, 313 (1996).

${ }^{49}$ J. Raabe, G. Tzvetkov, U. Flechsig, M. Böge, A. Jaggi, B. Sarafimov, M. G. C. Vernooij, T. Huthwelker, H. Ade, D. Kilcoyne, T. Tyliszczak, R. H. Fink, and C. Quitmann, Rev. Sci. Instrum. 79, 113704 (2008).

${ }^{50}$ M. Böge, U. Flechsig, J. Raabe, and T. Schilcher, in Proceedings of the Tenth European Particle Accelerator Conference EPAC 06, Edinburgh, Scotland (2006), p. 3610.

${ }^{51}$ G. Schütz, W. Wagner, W. Wilhelm, P. Kienle, R. Zeller, R. Frahm, and G. Materlik, Phys. Rev. Lett. 58, 737 (1987).
${ }^{52}$ M. R. Scheinfein: [http://llgmicro.home.mindspring.com].

${ }^{53}$ The saturation magnetizations were measured (Ref. 25) to $M_{\mathrm{s}}^{\mathrm{NiFe}}=$ $0.74 \times 10^{6} \mathrm{~A} / \mathrm{m}, M_{\mathrm{s}}^{\mathrm{NiFe}: \mathrm{Ne}}=0.67 \times 10^{6} \mathrm{~A} / \mathrm{m}, M_{\mathrm{s}}^{\mathrm{Co}}=1.27 \times 10^{6}$ $\mathrm{A} / \mathrm{m}$, and $M_{\mathrm{s}}^{\mathrm{Co}: \mathrm{Ne}}=1.16 \times 10^{6} \mathrm{~A} / \mathrm{m}$. The intrinsic anisotropies were set to zero and standard exchange constants $(A)$ were used: $A^{\mathrm{NiFe}}=1.05 \times 10^{-11} \mathrm{~J} / \mathrm{m}, A^{\mathrm{Co}}=3.05 \times 10^{-11} \mathrm{~J} / \mathrm{m}$. For making the simulations comparable, vacuum permeability was assumed for the interlayer.

${ }^{54}$ T. Gilbert, IEEE Trans. Magn. 40, 3443 (2004).

${ }^{55}$ K. Y. Guslienko, V. Novosad, Y. Otani, H. Shima, and K. Fukamichi, Appl. Phys. Lett. 78, 3848 (2001).

${ }^{56}$ Micromagnetic simulations reveal that for PDC samples, the AP configuration exhibits an intrinsic relative core displacement due to repulsive dipolar interaction (not shown), whereas for the $\mathrm{P}$ state the cores are expected to attract each other (Ref. 31). However, this effect is small compared to the displacements and fields investigated, and in IEC samples it is suppressed (Ref. 25).

${ }^{57}$ N. Martin, N.-C. Bigall, I. Mönch, T. Gemming, A. Eychmüller, R. Mattheis, R. Schäfer, L. Schultz, and J. McCord, Adv. Funct. Mater. 21, 891 (2011).

${ }^{58}$ Simulation parameters: $M_{\mathrm{eff}}^{\mathrm{FM}}=0.92 \times 10^{6} \mathrm{~A} / \mathrm{m}, M_{\mathrm{eff}}^{\mathrm{AF}}=0.33 \times$ $10^{6} \mathrm{~A} / \mathrm{m}, A=2.05 \times 10^{-11} \mathrm{~J} / \mathrm{m}$. In accordance with Eq. (5), the effective $c$ initialization corresponded to that of the Co layer. 